\title{
INVESTIGACION
}

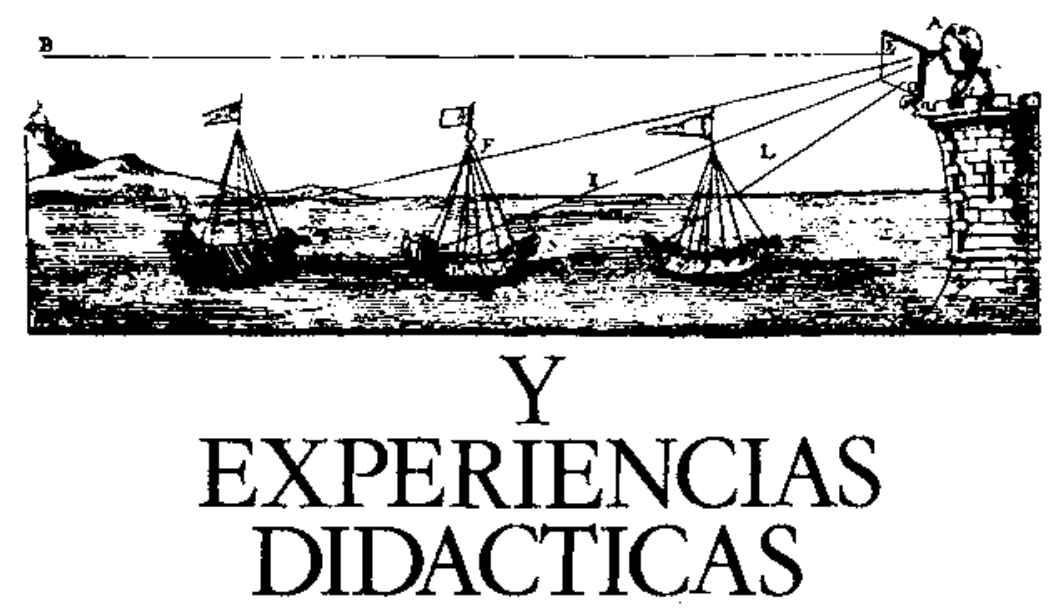

\section{ENFOQUES DE INVESTIGACIÓN EN PROBLEMAS VERBALES ARITMÉTICOS ADITIVOS}

CASTRO MARTÍNEZ, E., RICO ROMERO, L. y GIL CUADRA, F.

Departamento de Didáctica de ta Matemática. Universidad de Granada.

\section{SUMMARY}

A main field in the current research in Mathematics Education is the work with arithmetical word problem solving, which is both interesting and useful. There is ample previous work on this topic and it has received a very systematic treatment from different focuses. Researchers getting involved in this field need to know previous works and current focuses to clarify their research goals. In this study we offer a review about previous research done on difficulties with arithmetical word problems.

\section{INTRODUCCIÓN}

La Aritmética como materia escolar se ha justificado históricamente unas veces por el interés de la materia en sí misma, otras por la disciplina mental derivada de su estudio $\mathrm{y}$, sobre todo, por su utilidad práctica. A este respecto señala Reed que «el fin de la aritmética (escolar) es desarrollar el conocimiento de las relaciones cuantitativas y la habilidad para resolver los problemas relativos a los números y la cantidad que se presentan en 
las transacciones ordinarias de la vida... Naturalmente, la aritmética no se estudia únicamente por saber aritmética, ni por la disciplina mental derivada de su estudio, sino por su aplicación en ciertas actividades esenciales de la vida. Mientras más conocimiento tiene el alumno de estas actividades y de los objetos con que trata la aritmética, más significativa y valiosa resulta la materia.» (Reed 1949, p. 301).

Los problemas aritméticos verbales se incluyen en ei currículo escolar con la finalidad, entre otras, de facilitar al alumno este acercamiento entre Aritmética y realidad, entre Aritmética y aplicaciones a la vida real, que hacen más significativo y valioso su estudio.

En los problemas de expresión o formato verbal la información viene dada mediante un tex to que consta de una o varias frases. A los problemas aritméticos cuya expresión o enunciado es verbal se les llama problemas aritméticos verbales (PAEV).

Desde comienzos de este siglo, los investigadores han estado explorando las dificultades asociadas con la resolución de problemas enunciados verbaimente. El estudio de Kilpatrick (1991) presenta un magnífico resumen de las investigaciones de principio de siglo en Educación Matemática, con especiał mención de la resolución de problemas aritméticos. Kilpatrick subraya que el Report of the National Society for the Study of Education (Whipple 1930) supuso un momento de cambio para el tratamiento de la Aritmética, en particular por la crítica realizada al predominio en el aprendizaje de destrezas y rutinas y por la defensa que hace en favor de una mayor atención a los estudios sobre resolución de problemas y pensamiento cuantitativo.

También Strech (1941), en una revisión realizada sobre investigaciones y estudios relativos a la enseñanza y aprendizaje de la Aritmética, ofrece una selección de 100 trabajos de los realizados en USA hasta la fecha basándose en tres criterios: validez de las conclusiones, excelencia de la técnica empleada, y efecto de los resultados sobre la práctica educativa. Aunque hay una cantidad considerable de informes y estudios de carácter general, que afectan a todos los tópicos de la Aritmética, 18 de los trabajos incluidos en la lista hacen referencia explícita en su título a la resolución de problemas. Por tanto, la resolución de problemas en Aritmética es un campo de investigación presente desde los comienzos de la Educación Matemática como área científica de conocimiento.

En este artículo pretendemos dar una perspectiva de cuáles han sido los principales enfoques con los que se ha abordado el estudio de las dificultades en resolución de problemas aritméticos.

\section{ENFOQUES DE LAS INVESTIGACIONES}

En las investigaciones sobre resolución de problemas verbales y detección del nivel de dificultad que presen- tan para los escolares hemos encontrado cuatro enfoques fundamentaies:

1) el enfoque lingüístico,

2) el enfoque de variables estructurales,

3) el enfoque de sentencias abiertas, y

4) el enfoque semántico.

\section{EJ enfoque lingüístico}

En muchas de las investigaciones se ha prestado especial interés al papel que juega el lenguaje y las cuestiones asociadas en la resolución de este tipo de problemas. En las investigaciones que han tratado las dificultades de resolución de problemas en función del lenguaje, podemos distinguir las que se centraron en:

a) la habilidad lectora,

b) la legibilidad de textos, $y$

c) factores linguísticos.

\subsection{Habilidad lectora}

Las primeras investigaciones sobre resolución de pro* blemas aritméticos verbales se centraron fundamentaimente en el papel que desempeña la lectura. Reed cita las investigaciones hechas por Lessenger, Wilson, Monro: y Engelhart, en las que se pusieron de manifiesto que si se mejora la habilidad para leer, aumenta la habilidad para resolver problemas verbales. Lessenger, en 1925 , constató el hecho de que a un adelanto logrado en lectura correspondía un progreso igual en el cálculo. Wilson, en 1922, ensayó un método para mejorar la comprensión de los problemas verbales que consistía en: primero, hacer una serie de preguntas conducentes a aclarar el significado del problema; segundo, hacer una composición tomando el problema como tema; y tercero, dramatizar el problema o presentar la solución por medio de una pantomima. Esta práctica resultó ser favorabie. Monroe y Engelhart, en 1933, también obtuvieron que la enseñanza sistemática en la lectura de problemas verbales mejora el rendimiento, sobre todo de los alumnos retrasados. Estos investigadores ejercitaban a los alumnos en definir términos, indicar procesos, formular problemas semejantes y esquematizar las condiciones de los problemas. Obtuvieron sólo una pequeña diferencia en favor del grupo experimental, lo que según Reed pudo ser debido a dos razones: primero, la diferencia entre los métodos de instrucción fue muy pequeña; y, segundo, mejorar la habilidad para la lectura más allá de ciertos límites tiene poco valor sobre la habilidad para resolver problemas (Reed 1949).

En investigaciones posteriores que siguen este enfoque se acepta ya como hipótesis que existe comelación entre la habillidad lectora y el exito en resolución de problemas verbales. Muchas de ellas concluyen dando un coefi. ciente de correlación significativo (Aiken 1971).

La dificultad del problema verbal se identifica fundamentalmente en este enfoque con la dificultad de aprendizaje de una nueva lengua. Por ello, se proponen métodos de enseñar a resolver problemas verbales que enfatizan 
la traducción del enunciado del problema a una relación numérica. En el método de Dahmus, por ejemplo, los estudiantes primero aprenden cómo convertir enunciados del idioma inglés en enunciados matemáticos y a continuación resolver las ecuaciones o sistemas de ecuaciones a que dan lugar. Dahmus destaca que su método es especialmente efectivo con los alumnos retardados (Dahmus 1970).

\subsection{Legibilidad}

En el período comprendidoentre 1950 y 1980 gran número de investigadores se han dedicado a investigar el nivel de legibilidad de los libros de texto de Matemáticas y han intentado aplicar fórmulas ideadas para medir el nivel de legibilidad de textos corrientes (narrativos u otros) a los textos que aparecen en los libros de Matemáticas y particularmente a los problemas verbales (Barnett 1980).

Dentro de este enfoque, Kane (1970) afirma que es inapropiada la aplicación de fórmulas de legibilidad ideadas para el lenguaje natural a textos matemáticos. Kane afirma que los textos de Matemáticas están escritos en más de un lenguaje: la lengua materna, La notación indoarábiga đe los números, el sistema de notación algebraico, el lenguaje del cálculo de proposiciones, etc. Estos lenguajes aparecen mezclados en mayor o menor medida de unos libros a otros y de unas unidades didácticas a otras. La lengua materna se ve complementada con expresiones que son traducciones de algunos de los lenguajes adicionales que usa la Matemática. Por ejemplo, frases tales como «si y sólo si», «si ...entonces», «A y B»... son traducciones del lenguaje del cálculo proposicional. El significado preciso de estas traducciones deberá ser comprendido por el lector antes de que aparezcan empleadas en un texto, si queremos que entienda el significado del mismo. Kane sostiene la idea de que la resolución de problemas verbales es una mera traducción entre lenguajes.

Más recientemente Paul, Nibbelink y Hoover (1986) hacen un estudio para comprobar si las fórmulas de legibilidad proporcionan información fiable que permita decidir cuándo determinados problemas verbales son apropiados para alumnos de un nivel dado. Las fórmulas de legibilidad que se emplean en esta investigación son la Fórmula de Dale-Chall y la Fórmula de Spache. Los resultados de este estudio no sostienen la hipótesis de que las fórmulas de legibilidad sean útiles para predecir la dificultad de los problemas verbales.

Otros investigadores (Moyer y otros 1984a, 1984b) han estudiado la dificultad de los problemas en función de formatos distintos y contrapuestos que se supone que tienen distinto grado de legibilidad: problemas verbales versus telegráficos, problemas con dibujos versus ver. bales, versus telegráficos.

\section{El enfoque de variables estructurales}

El concepto variable de tarea es utilizado por Kilpatrick (1978) y por otros autores posteriores como Goldin y
McClintock (1980). En estos trabajos se refieren a ellas como características de los enunciados de los problemas que asumen un valor particular dentro de un conjunto de valores posibles. Un tipo de variable de tarea correspondiente a la estructura superficial del problema son las variables sintácticas (Goldin y McClintock 1980). En el enfoque adoptado para estudiar la influencia de las variables de estructura superficial sobre la dificultad del problema, expresada en porcentaje de éxitos, se considera el problema aritmético verbal como un ente independiente del resolutor que puede ser descrito mediante un número finito de características discretas, a las que Nesher (1976) llama variables estructurales. Detectar si cada una de estas variables estructurales tiene influencia significativa o no sobre la dificultad del problema es un objetivo general de este grupo de investigaciones. Sin embargo, no todas persiguen el mismo fin, ni adoptan la misma metodología. Como señalan Puig y Cerdán (1989), los estudios realizados bajo este enfoque pueden clasificarse en dos categorías. Los que pretenden pređecir la dificultad del problema en función de todas las variables estructurales que tengan influencia significativa sobre esa dificultad, y los estudios que han tratado de determ:nar si una variable en particular afectaba de forma significativa al nivel de dificultad expresadoen porcentajes, controlando el resto de las variables. Al primer enfoque lo vamos a Ilamar análisis global de variables, y al segundo, análisis parcial de variables.

\subsection{Análisis global}

Los investigadores de la primera categoria tratan de expresar la dificultad del problema como la suma de las dificultades aportadas por cada una de las variables estructurales, tales como la longitud del problema, la complejidad gramatical de sus oraciones, la operación con la que resuelve el problema o el orden en el que aparece la información. Los trabajos más significativos dentro de esta categoría del enfoque de variables estructurales son los realizados en el Institute for Mathematical Studies in the Social Sciences de la Stanford University por un grupo de investigadores bajo la dirección de $\mathrm{P}$. Suppes. Además de trabajar en la construcción de un programa de enseñanza por ordenador para aprender a resolver problemas aritméticos verbales, intentan identificar en sus estudios variables estructurales que permitan predecir la dificultad de los problemas aritméticos verbales para los estudiantes. (Suppes, Loftus y Jerman 1969 , Jerman y Rees 1972, Jerman 1973, Searle, Lorton y Suppes 1974).

Utilizando la técnica estadística del análisis de regresión lineal pretendían encontrar una recta de regresión que permitiera poder predecir la dificultad de un problema aritmético verbal en función de las variables discretas que tuvieran efecto significativo sobre la dificultad.

Una așunción fundamental de esta aproximación, como lo expresan Jerman y Rees (1972), es que la estructura (superficial) de los problemas aritméticos por símisma, para un gran número de mediciones determina su nivel de dificultad. El grupo es consciente de que los factores de aptitud-interacción del estudiante frente al problema 
son importantes, pero hasta que no haya una teoría que describa convincentemente la naturaleza de tales factores, los componentes del grupo opinar que mediante el análisis de tipo estructural resultará un camino fructífero para la investigación y desarrollo del currículo. La pretensión final es poder formular un conjunto claro de reglas para generar conjuntos de problemas ariłméticos de un nivel especúfico de dificultad. «Los diseñadores de currículo estarían entonces en una mejor posición para controlar el nivel de dificultad cuando preparan materiales para la enseñanza» (Jerman y Rees 1972).

El enfoque de variables estructurales tiene por referencia básica el trabajo de Suppes, Loftus y Jerman (1969). En esta investigacion las variables que se anatizan como posibles factores que afectan a la dificultad de un problema son: operaciones, pasos, longitud, secuencia, pista verbal y conversion.

La distinción entre la variable «operaciones» y "pasos» queda reflejada claramente en el siguiente ejemplo: Un problema que pida hallar la media de 11 números tiene 11 pasos y 2 operaciones.

Para reafirmar la importancia de la variable longitud, Suppes, Loftus y Jerman citan investigaciones sobre la adquisición del lenguaje en las que se pone de manifiesto el desarrollo gradual de los niños en la comprensión de sentencias de menor a mayor número de palabras y la dificultad que tienen éstos para retener sentencias.

Las palabras que consideraron en este trabajo como pistas verbales son: para la adición: «y»; para la substracción: «menos» o un comparativo; para la multiplicación: «cada uno»; para la división: «promedio» o «cada uno» apareciendo en la pregunta.

La variable «conversión» se definió para el caso en que los estudiantes tuvieran que recordar de memoria hechos tales como que $\ll 1$ semana $=7$ días».

Se utilizaron oraciones tales como «Cuál fue su ventaja neta en metros», que dejan a los estudiantes sin Ia menor idea de qué operación usar. Esto sugiere la idea de que la presencia o ausencia de una palabra clave puede ser un potente indicativo de la dificuitad del ítem.

Los resuitados que obtuvieron en esta investigación muestrart que sólo tres de las variables estudiadas -operaciones, secuencia y conversión de unidades-son significativas. El modelo de regresión lineal en esta investigación da cuenta del $45 \%$ de la varianza de la probabilidad de que la respuesta sea correcta.

Una constante en los sucesivos trabajos de este enfoque es que los investigadores tratan de identificar nuevas variables que añadir a las investigadas anteriormente. En concreto, Bárbara Searle (Jerman y Rees 1972) utiliza los datos obtenidos en la investigación anterior para estudiar un conjunto de 13 variables. Dichas variables habían sido tratadas en un contexto CAI donde los estudiantes sólo tuvieron que indicar las operaciones que era necesario realizar, pero no efectuar los cálculos. Jerman y Rees (1972) consideran de interés examinar el efecto de estas mismas variables cuando se aplican a problemas resueltos con lápiz y papel. Ello les llevó a definir otras variables e incrementar hasta 22 la lista de las variables consideradas por Searle. En las conclusiones de este trabajo, Jerman y Rees afírman que sólo cinco de las variables consideradas dan cuenta aproximadamente del $87 \%$ de la varianza de la probabilidad de que la respuesta sea correcta. La que más contaba fue la varia. ble multiplicación con el $32 \%$, seguida por ia variable división con el $23 \%$, la variable Iongitud del problema conel $21 \%$, la variable distractor con el $11 \%$, y finalmente la variable adición-sustracción con el $1 \%$.

El proceso de identificar y recopilar variables estructurales en los enunciados de los problemas aritméticos verbales es llevado a su mayor refinamiento por Jerman y Mirman (1974). Establecen dos grandes bloques: va riables linguísticas y variabies computacionales. Las variables computacionales que consideran en este traba* jo son seis de las ya empleadas en los trabajos anteriores (conversión, memoria, longitud, operaciones, NOMC2 y cociente), mientras que las lingüísticas son una recopilación de variables ya utilizadas por otros investigadores a las que añaden otras nuevas no investigadas anteriormente y que agrupan en siete categorías: 1) longitud, 2) partes de la oración, 3) palabras, 4) números, 5) oraciones, 6) elementos de las oraciones, y 7) puntuación* símbolos-caracteres.

\subsection{Análisis parcial de variables}

La segunda categoría de investigaciones analizá el efecto de determinados factores sobre el rendimiento de los sujetos. El análisis de regresión no es la técnica estadís. tica obligada, como ocurría en el caso anterior, y se utilizan técnicas estadísticas altemativas comoel análisis de la varianza. Éste es el caso de Linville (1976), que estudia la habilidad de resolver problemas verbales en función de la dificuitad del vocabulario y de la sintaxis, o el de Nesher (1976), que estudia la influencia de las variables número de pasos, presencia o no de información superffua y presencia o no de palabras clave.

\section{El enfoque de sentencias abiertas}

En este enfoque las investigaciones se caracterizan por clasificar los problemas aritméticos a partir de las sen. tencias abiertas que subyacen en el problema, con el ánimo de distinguir entre ellas distintos niveles de rendimiento o distintas estrategias en escolares de los primeros niveles. Las investigaciones iniciales se hacen sólo sobre dificultades relativas de sentencias numéricas simples de adición y sustracción; así por ejemplo, Wea* ver (1971, 1972, 1973) investiga el nivel de rendimiento de escolares de $1^{\circ}, 2^{\circ}$ y $3^{\circ}$ grado, al resolver determinadas sentencias abiertas de adición y sustracción. Compara el nivel de rendimiento: medido en porcentajes de aciertos, de los tres grados, el de sentencias análogas de adición y substracción ([ ] $+a=b,[]-a=b)$ el de una sentencia abierta y su simétrica $(a-[]=b, b=a-[])$, el de sentencias cuya solución es un número natural, con otras que carecen de ella, y las diferencias debidas a la posición de 
La incógnita, es decir, si el dato desconocidoes uno de los dos que se componen o bien lo es el resultado.

Posteriormente se incorporan nuevos elementos en las investigaciones sobre sentencias abiertas. Así, Grows (1972) estudia el rendimiento en sentencias abiertas de adición y sustracción en función del sexo, y además investiga ia influencia sobre el rendimiento en una determinada sentencia si ésta está o no acompañada de un enunciado verbal en el que subyace dicha sentencia.

En posteriores trabajos se analizan otros aspectos en función de las sentencias abiertas, así, Grows (1974) utiliza la técnica de entrevista individual para aplicar un test elaborado con sentencias abiertas e investigar los métodos (estrategias) que los niños utilizan para resolverlas. La finalidad del estudio fue:

1) Identificar los métodos de solución empleados por los niños y determinar su frecuencia de uso.

2) Indagar la extensión con la que diferentes métodos de solución son usados por los niños para resolver sentencias abiertas similares.

3) Examinar la relación entre el número de soluciones correctas y tipos de métodos empleados.

El estudio realizado por Lindvall e Ibarra (1980) con niños de $1^{9}$ y $2^{0}$ de diferentes comunidades trata de la identificación de estrategias incorrectas y la frecuencia con la que aparecen. El estudio implica la resolución con lápiz y pápel de sentencias abiertas, la habilidad para leerlas, la resolución de problemas verbales en los que estaban implicadas dichas sentencias y la influencia de material manipulativo para demostrar las soluciones obtenidas.

Así mismo, Hiebert (1982) investiga las estrategias que siguen los escolares de primer grado al resolver problemas verbales de adición y sustracción con datos menores de 10. Los problemas utilizados fueron seis, pertenecientes a la estructura semántica de cambio, correspondiendo tres a unión y tres a separación. En cada caso las tres posibilidades surgen al variar sistemáticamente la posin ción de la incógnita. A los niños, se les daba la posibili. dad de poder utilizar material manipulativo (cubos pequeños) como ayuda para resolver el problema. Los resultados obtenidos ponen de manifiesto que la posición de la incógnita tiene una influencia sustancial en la conducta que siguen los niños.

En un trabajo nuestro sobre resolución de problemas verbales aritméticos de comparación de estructura mul* tiplicativa (Castro, Rico, Batanero y Castro 1991) pone. mos de manifiesto también que la posición de la incóg. nita tiene efecto significativo sobre el nivel de dificultad, medido en porcentajes de aciertos, con niños de 10 a 12 años.

Como conclusión de estos estudios podemos decir que dentro de las investigaciones previas sobre dificultades en problemas aritméticos verbales de adición y sustracción, la variable posición de la incógnita en la sentencia abierta subyacente en el problema, cuyos valores representan la identidad de la cantidad desconocida, se ha mostrado como una de las variables más interesantes y que con más claridad ha establecido diferencias de dificultad entre problemas.

\section{El enfoque semántico}

Para resolver un problema aritmético enunciado verbal. mente (PAEV) es necesario conocer el significado del texto en el que está enunciado el problema. En un PAEV todas las palabras del texto de su enunciado no juegan el mismo papel desde el punto de vista de su resolución. Se pueden distinguir, como hacen Puig y Cerdán (1989), «las que desempeñan algún papel en la elección de la operación y las que no desempeñan papel alguno. El papel de estas últimas suele limitarse a conectar el enunciado del problema con la realidad o a delimitar el contexto del problema». Las palabras -o grupos de palabras-que influyen en la elección de la operación que hay que realizar se denominan palabras clave.

En las investigaciones de PAEV que se han centrado en el estudio del significado han existido dos tendencias claramente diferenciadas: las que los han estudiado en base a significaciones parciales del texto y las que lo hacen globalmente, considerando el texto del problema como un todo.

Dentro de las investigaciones sobre significaciones par" ciales se han utilizado variables de contenido semántico con la creencia de que la resolución correcta de un PAEV depende de la asociación adecuada entre una «palabra clave» y la operación que «representa». Por el contrario, el análisis global, aunque no margina el papel de las palabras clave a la hora de resolver el problema -éstas ya no son los únicos determinantes en la interpretación del significado del problema-hace intervenir otros factores que convierten en relativa su aportación. Las investigaciones que se centran en el análisis global del problema son investigaciones de tipo estructural, en las que se admite que el resolutor utiliza determinados esquemas conceptuales para comprender el significado del texto del problema. Puig y Cerdán (1989) califican de ingenuo el enfoque del análisis basado en palabras clave y mantienen que el enfoque global es el más adecuado y el que tiene mayor actualidad.

\subsection{Palabras clave}

Las variables de contenido semántico describen los significados de palabras y expresiones matemáticas que aparecen en el enunciado del problema y se supone que tienen una influencia decisiva a la hora de elegir la operación con la que solucionarlo. Webb (1980) considera que estas variables de contenido semántico se han utilizadoen la investigación para «describir los significados del lenguaje natural y del lenguaje técnico que pueden ínfluir en el nivel de dificultad en la resolución de problemas", y las divide en dos categorías: palabras clave y vocabulario matemático. 
Para Webb la razón de ser de estas variables en la investigación es la siguiente: Durante su período de escolarización, los alumnos deben resolver una gran cantidad de problemas que están enunciados verbalmente, es decir, que están expresados en el lenguaje natural. Para resolver estos problemas verbales, el sujeto debe comprender el enunciado verbal y traducirlo a expresiones matemáticas que representen la estructura del problema.

La investigación sobre palabras clave recibió un fuerte impulso con los trabajos del Grupo de Stanford sobre variables estructurales. Así Suppes y otros (1969), intentando determinar factores que afecten a la dificultad de un problema, investigaron un conjunto de seis variables, una de las cuales es la presencia o no de palabras clave en los enunciados de los problemas. Como hemos señalado anteriormente, las palabras clave que utilizaron fueron "y» para la suma, «menos» o un comparativo para la resta, «cada uno» para la multiplicación, «promedio» o «cada uno» colocado en la sentencia interrogativa del enunciado del problema para la división. Los resultados obtenidos por Suppes y sus colaboradores muestran que no hallaron efectos significativos de regresión de esta variable para la dificultad de los problemas, es decir, que la presencia o no de palabras clave no explicaba una proporción suficiente de la varianza observada.

Jerman (1973) prestó especial atención a las palabras clave y definió varias variables a partir de ellas, tratando de dilucidar si la presencia o no de palabras clave representaba una mayor o menor dificultad en el problema. No obtuvo resuliados significativos, salvo cuando actúan como distractores, de los cuales la ausencia o presencia de una palabra clave en un problema afecta a su nivel de dificultad.

Nesher y Teubal (1975), desde un enfoque del procesamiento de la información, estudiaron la influencia de palabras clave en el proceso de traducción, desde la formulación verbal del problema hasta la expresión matemática del mismo. En concreto, investigaron en problemas que se pueden resolver con sumas y restas, si la elección de una de estas operaciones para solucionar el problema está influida por la presencia de una palabra clave en el enunciado del mismo. Las palabras clave aparecen unas veces empleadas como pistas verbales de la operación a realizar y otras como distractores. Los resultados mostraron un menor rendimiento de los sujetos en los problemas donde las palabras clave actuaban como distractores. La conciusión que sacó Nesher de este trabajo es que «si una palabra no tiene un unívoco y definitivo significado en todo problema verbal, no puede ser usada como palabra clave. Hay que hacer comprender las relaciones matemáticas subyacentes que están expresadas en la formulación verbal» (Nesher 1976).

Nesher (1976) estudió la influencia de tres variables en la dificultad de problemas aritméticos, una de ellas son las «palabras clave». En esta investigación las palabras clave no actúan como distractores. Los datos obtenidos fueron tratados estadísticamente mediante el análisis de varianza. De este análisis resultó que la variable «pala- bras clave" no tenía efecto significativo sobre la dificultad de resolución de los problemas estudiados por ella.

En una investigación realizada por nuestro grupo (González et al. 1985) se estudió el papel de los verbos de acción como determinantes de la operación que hay que elegir para resolver problemas aritméticos simples y se realizó una clasificación de verbos por operación.

Las investigaciones sobre vocabulario matemático se basan en el supuesto de que existe distinción entre el significado de una palabra usada en el lenguaje usual y la misma palabra usada con significado maternático. Investigado. res como Kane (1970) sostienen que la lectura de textos matemáticos requiere una habilidad especial diferente de la requerida para leer textos ordinarios, por lo que necesita de una particular instrucción.

\subsection{El enfoque de esquemas mentales}

Los enfoques teóricos más recientes utilizados en las investigaciones sobre resolución đe problemas aritméticos verbales se basan en el análisis del esquema mental que utiliza el resolutor cuando resuelve un problema verbal. Estos enfoques de esquemas mentales se enrai. zan en la tradición psicológica de Bartlett, centrada en la cuestión de carácter cognitivo sobre cómo se organiza el conocimiento verbal en la cabeza de las personas. Son pues enfoques cognitivos de la resolución de problemas verbales que consisten en intentar analizar este tipo de conocimiento verbal en sus partes e indicar la estructura en la que se enlazan éstas. Por ello, en este enfoque, una categoría de problemas verbales es un modelo estructural de conocimiento verbal de una persona que consta generalmente de elementos y relaciones entre esos elementos.

En la segunda mitad de la década de los setenta y primeros años de los ochenta, varios investigadores estuvieron empleando, por separado, el enfoque de esquemas mentales en sus investigaciones sobre problemas aritméticos verbales simples de estructura aditiva, $y$ clasificaron los problemas en categorías semánticas similares. Entre ellos destacan las aportaciones de Vergnaud, Greeno y Heller, Carpenter y Moser, y Nesher.

En este período las investigaciones sobre problemas aritméticos se dividen para su estudio en dos campos: el campo de la estructura aditiva y el campo de la estructura multiplicativa. Los problemas de estructura aditiva son aquéllos cuyas soluciones implican solamente sumas y restas. Mientras que los problemas de estructura multiplicativa implican sólo multiplicaciones y divisiones. Estos dos campos se estudian por separado. A continuación vamos a tratar las investigaciones realizadas sobre la estructura aditiva.

Dentro de este enfoque estructural se pueden distinguir dos corrientes:

a) la corriente impulsada por el psicólogo francés Gerard Vergnaud, que utiliza el cálculo relacional como con- 
cepto esencial para comprender el funcionamiento $\operatorname{cog}-$ nitivo del sujeto; y

b) la corriente basada en las categorías semánticas (unir, separar, etc.) de los problemas.

\subsubsection{El cálculo relacional}

Para Vergnaud el estudio clásico de los problemas de Aritmética elemental en base a la diferenciación según las operaciones de adición, substracción, multiplicación y división crea dificultades y no caracteriza ciertas relaciones numéricas que aparecen en los enunciados de los problemas. Por ello, distingue, basándose en la noción de campo conceptual, entre el campo conceptual de las estructuras aditivas y el de las estructuras multiplicativas.

Una noción clave en el enfoque de Vergnaud es si los datos del problema expresan, o no, una acción que se desarrolla en el tiempo. Para realizar esta distinción utiliza los conceptos de estado (medida) y de operador (transformación).

En base a estas nociones realiza una categorización de los problemas aritméticos de estructura aditiva en cinco grandes tipos (Vergnaud y Durand 1976):

1) dos medidas que se componen en una tercera,

2) una transformación que opera sobre una medida para dar una medida,

\section{3) dos transformaciones que se componen en una tercera,}

4) una transformación que opera sobre un estado relativo para dar otro estado relativo,

5) dos estados relativos que se componen en un tercero.

Basándose en esta clasificación trató de establecer una jerarquía por el nivel de dificultađ que los diversos tipos de problemas plantean a los niños. Así, obtuvo que los problemas de la categoría tercera (composición de transformaciones) son más difíciles que los de la categoría segunda (transformación entre estados).

Vergnaud considera además que cada una de estas categorías da lugar a varias clases de problemas de diferente grado de dificultad según la identidad -posición- de la incógnita, la dimensión absoluta o relativa de los números, que sean enteros o decimales, según el orden de presentación, la estructuración sintáctica de los enunciados, etc.

En un trabajo posterior, este investigador (Vergnaud 1982) añade otra categoría a las definidas en 1976 y las formula del siguiente modo: 1) composición de dos medidas, 2) transformación entre dos medidas, 3) relación estática entre dos medidas, 4) composición de dos transformaciones, 5) transformación entre dos relaciones estáticas, 6) composición de dos relaciones estáticas.
No obstante, hay que señalar que sus trabajos en estructuras aditivas han recibido menor atención que los que ha realizado en el campo de las estructuras multiplicativas. Puesto que en este artículo no vamos a tratar los enfoques utilizados en investigaciones sobre resolución de problemas verbales de estructura muitiplicativa, dejamos al matgen su aportación en este campo (Vergnaud 1983).

\subsubsection{Las categorías semánticas}

En un trabajo presentado por Heller y Greeno en 1978 sobre procesamiento semántico de los problemas verbales (Heller y Greeno 1979), estos autores distinguen tres esquemas que representan estructuras alternativas de información cuantitativa relativas a problemas de adición y substracción, a las que liaman Causa/Cambio, Combinación y Comparación. Este análisis semántico es coincidente con el realizado por Nesher y Katriel en 1978 (Nesher 1982), aunque utilizan un nombre distinto para algunas de las categorías.

Los problemas de Causa/Cambio son los que describen situaciones en los que algún evento cambia el valor de una cantidad. Por ejemplo: «Juan tiene 5 bolas, José le da 3 más» manifiesta un cambio en la cantidad de objetos poseidos por una persona como resultado de una acción. El esquema abstracto subyacente contiene una cantidad inicial, una acción que implica un cambio de valor, bien sea de aumento o de disminución, y una cantidad final resultante. La dirección del cambio así como la identidad de la cantidad desconocida determinan la operación matemática necesaria para resolver el problema. En función de estas dos variables hay seis posibles problemas de Causa/Cambio. Esta categoría es similar a la categoría que Vergnaud denomina «transformación entre dos medidas». Carpenter, Hiebert y Moser (1981) y Carpenter y Moser (1982) la denominan en estos trabajos previos problemas de "unión y de separación», y Nesher (1982) la denomina categoría «dinámica». Actualmente hay común acuerdo entre los investigadores en denominar a esta categoría problemas de estructura semántica de cambio.

Los problemas de combinación se basan en la relación estática existente entre un conjunto total y dos subconjuntos disjuntos cuya unión sea el conjunto total. Por ejemplo: «Juan tiene 4 caramelos. Ana tiene 5 caramelos. ¿Cuántos caramelos tienen entre los dos? ». Según la identidad de la cantidad desconocida hay dos tipos de problemas de combinación: se conoce el tamaño de los dos subconjuntos y se pide el tamaño de la unión, o se conoce uno de los subconjuntos y su unión y se pide el tamaño del otro subconjunto. Esta categoría coincide con la que Vergnaud llama «composición de dos medidas». Carpenter, Hiebert y Moser (1981), y Carpenter y Moser (1982) la llaman «parte-parte-todo». Nesher (1982) la llama categoría «estática». Actualmente esta categoría de problemas es comúnmente denominada problemas de estructura semántica de combinación.

EI tercer esquema, el de comparación, al igual que el de cambio, implica relaciones estáticas, es decir, relaciones 
en las que no hay implícita una acción. Los problemas de comparación implican la comparación de dos cantidades, una de los cuales es la cantidad referente y la otra, la comparada o referido. La tercera cantidad es la diferencia, o cantidad en la que la más grande excede a la otra. Por ejemplo: «María tiene tres cintas. Susana tiene 8 cintas. ¿Cuántas cintas tiene Susana más que María?». Según la đirección de la comparación (más que o menos que) y la cantidad desconocida (referente, referido o diferencia), surgen seis tipos de problemas de comparación. Esta categoría se corresponde con la que Vergnaud (1982) llama «relación estática entre medidas», Carpenter, Hiebert y Moser (1981), Carpenter y Moser (1982), y Nesher (1982) denominan también esta categoría como problemas de estructura semántica de comparación, que es la que se usa actualmente.

Carpenter, Hiebert y Moser (1981), Carpenter y Moser (1982 1983) consideran además la categoría de igualación, híbrida de las de cambio y comparación. Se trata de problemas en los que se demanda la acción que hay que realizar sobre una cantidad para hacerla igual a otra. Por ejemplo: «Juan tiene 50 pesetas. Antonio tiene 20 pesetás. ¿Cuántas tiene que perder Juan para tener tantas como Antonio?». Según que la acción a realizar se aplique sobre la cantidad mayor o menor y según cuál sea la identidad de la cantidad desconocida en la igualación, surgen seis tipos de problemas de igualación.

Las categorías semánticas se han aplicado a problemas simples de adición y substracción que se suponen que son apropiados para escolares de las primeros grados, en los que se utilizan sólo números naturales. En esto se diferencia del enfoque adoptado por Vergnaud, que trata de hacer una clasificación más exhaustiva en la que intenta englobar tipos de problemas en un período más amplio del currículo escolar, y no sólo de los primeros grados. Como consecuencia de elio, las categorías elaboradas por Vergnaud abarcan problemas aritméticos verbales con clases más amplias de números, como por ejemplo, los números enteros.

Durante la década de los ochenta, numerosos investigadores han estudiado la resolución de problemas de estructura aditiva en función de estas categorías de problemas. Buena prueba de ello la podemos encontrar en los trabajos de Carpenter, Hiebert y Moser (1981), Carpenter y Moser (1982, 1983, 1984), Riley, Greeno y Heller (1983), Nesher, Greeno y Riley (1982), Nesher (1982), Kintsch y Greeno (1985), De Corte y Verschaffel (1985, 1987), Bermejo y Rodríguez (1987a, 1987b). Gran número de ellos estudian el nivel de dificultad en función de las categorías semánticas y en función de la identidad de la cantidad desconocida.

Entre tos resultados sobre dificultades más difundidos dentro de este enfoque se encuentran los obtenidos por Riley y otros (1983) sobre las tres primeras categorías. El orden de dificultad que obtienen para las categorías es: cambio, combinación y comparación. Pero esta ordenación se complica cuando hacen intervenir la variable de identidad de la cantidad desconocida (posición de la incógnita). Dentro de una misma categoría de problemas hallan casos de bastante diferencia de dificultad según esta última variable:

- En la categoxía de cambio, los probiemas más fáciles son los de la cantidad fínal desconocida, bien sea la acción de aumento o de disminución, mientras que los más difíciles dentro de esa categoría corresponden a aquéllos en los que se desconoce la cantidad inicial.

- En problemas de combinación, los más fáciles son aquéllos en los que se pide hallar la cantidad total, frente a los que piden hallar alguna de las dos partes.

- En problemas de comparación, el tipo más dificil es cuando se pide hallar el referente.

Además de diferenciarse por el porcentaje de éxitos que obtienen los escolares de los primeros grados, las categorías semánticas provocan en los escolares procesos distintos de actuación (Carpenter y Moser 1983). Incluso De Corte y otros (1990) observan diferencias entre las categorías semánticas en función de los movimientos oculares de los niños durante la resolución de este tipo de problemas. Carpenter, Moser y Bebout (1988) estudian la incidencia de la estructura semántica de los problemas verbales aditivos de un paso sobre la sentencia abierta que le asignan los niños. Su conclusión es que «la estructura semántica de los problemas verbales influye directamente sobre la sentencia numérica que los niños escriben para representarlos» (p. 354). En otros trabajos dirigidos por Carpenter, por ejempio, en Bebout (1990) y Carey (1991), dentro de las categorías semánticas, se utilizan las sentencias abiertas para investigar las dificultades que tienen los niños en representar estos problemas simbólicamente.

\section{CONCLUSIONES}

En las investigaciones revisadas hemos querido resaltar la perspectiva, el planteamiento, con el que se ha abordado el estudio de las dificultades en problemas aritméticos verbales y subrayar algunos de los logros que en base a ellos se han obtenido. La utilidad de este trabajo es doble. Primero, es útil al investigador que quiere injciarse en este campo. La máxima "conocer el pasado para no repetirlo inútilmentes en un ciclo que nos impediría avanzar en el conocimiento es aplicable en este caso. Segundo, es útil al educador conocer cuáles son las dificultades que le puede plantear a los alumnos este tipo de problemas y posibles interpretaciones de ellas, pues este conocimiento le permitirá atacarlas con base científica.

Desde las primeras investigaciones hemos podido observar la gran atención que han recibido las variables lingüísticas como causa de las dificultades, y los numerosos intentos realizados por proponer y ensayar métodos que los solucionaran. Así mismo, se puede percibir el progresivo avance en el control de aquellas variables de tarea que se habían mostrado significativas en las investigaciones previas. De unas primeras investigaciones donde la única distinción era la de ser problema arit- 
mético y, por tanto, podían aparecer arbitrariamente en su resolución sumas, restas, multiplicaciones y divisiones, se pasa a tener en cuenta variables tales como: si el problema requiere una o más operaciones, si el problema requiere de uno o más pasos para su resolución, si el problema es de estructura aditiva o multiplicativa, cuál es la posición de la incógnita y cuál es la estructura o el esquema semántico de los enunciados. El enfoque de variables estructurales puso de manifiesto la influencia de determinadas variables sintácticas sobre la dificultad de resolución de los problemas aritméticos verbales, pero las investigaciones posteriores, basadas en esquemas, han puesto de manifiesto que la estructura semántica es una componente con mayor peso que la sintaxis a la hora de determinar la dificultad del problema. Las investigaciones más actuales parten ya de una clasificación de los problemas en estructuras semánticas y analizan la posible influencia de otros aspectos. Así, por ejemplo, Fayol y Abdi (1986), utilizando el esquema de clasificación de problemas de Vergnaud, estudiaron la influencia de la formulación verbal del problema manteniendo constante su estructura relacional. Otros autores también han estudiado la influencia del estilo del tex to sobre la dificultad de problemas pertenecientes a la misma categoría semántica, éste es el caso, por ejemplo, de De Corte, Verschaffel y De Win (1985). Los resultados obtenidos por estas investigaciones han conducido a los investigadores actuales a establecer que las diferencias de nivel de ejecución encontradas entre los niños cuando resuelven problemas aritméticos verbales simples de estructura aditiva se explican fundamentalmente en función de tres factores: la estructura semántica, el lugar ocupado por la incógnita y la formulación verbal.

Se observa también una evolución global de la perspectiva bajo ta que se realizan las investigaciones. Las primeras investigaciones están inmersas en una dinámica de proceso-producto en las que se postulaba que simplemente una mejora en los métodos de enseñanza mejoraría los resultados de los niños. Estas investigaciones predominan en la etapa inicial y perviven hasta la década de los sesenta. Si bien en esta fase se realizan investigaciones que se centran en determinar factores influyentes en Ia dificultad de los problemas, es a partir de esta década cuando proliferan investigaciones mediacionales que tratan de aislar factores de dificultad específicos que ocupan un Iugar intermedio en el proceso enseñanzaaprendizaje. Estas últimas investigaciones no se ocupan del problema de qué método es más efectivo para enseña a resolver problemas verbales, sino de cómo obtienen los resolutores los significados globales de los enunciados de los problemas. Por tanto, las consecuencias de tipo práctico que el educador puede sacar de estas últimas investigaciones no pueden ser de típo metodológico, sino de tipo puntual, que pueden tener cabida en una enseñanza diagnóstica.

La actitud del profesor ante el fracaso de sus alumnos en un problema verbal debe evolucionar desde los planteamientos simplistas de que todos los problemas que se resuelven con una misma operación tienen todos la misma dificultad, hasta aceptar que puede ser necesario para determinados alumnos la instrucción específica en problemas de distinta estructura semántica, los cuales, aunque se resuelvan con una misma operación, presentan un nivel de dificultad distinto. Tratar de hacer comprender el significado global de los problemas pertenecientes a distintas categorías semánticas antes que seguir una instrucción basada en el análisis de las palabras clave, el vocabulario o la sintaxis del problema son recomendaciones ampliamente aceptadas por los investigadores en este campo.

\section{REFERENCIAS BIBLIOGRÁFICAS}

AIKEN, L. R., Jr, 1971 . Verbal factors and mathematics learning: A review of research, Journal for Reseach in Mathematics Education, 2, pp. 304-313.

BARNETT, J, 1980. The study of syntax variables, en G.A. Goldin y C.E. McClintock (eds.), Task variables in mathematical problem solving, pp. 23-68. (The Franklin Institute Press.: Philadelphia, Pensilvania).

BERMEJO, V. y RODRÍGUEZ, P., 1987a. Estructura semántica y estrategias infantiles en la solución de problemas verbales de adición, Infancia y Aprendizaje, 39-40, pp. 71-81.

BERMEJO, V.y RODRIGUEZ,P., 1987b. Análisis de los factores incidentes en la solución de problemas de adicion: su estructura semántica, formulación y lugar de la incógnita, Enseñanza de las Ciencias, número extra, pp. 332-333.

BEBOUT, H.C.,1990. Children's symbolic representation of addition and subtraction word problems, Journalfor Research in Mathematics Education, 21, pp. 123+131.

CAREY, D. A., 1991. Number sentences: Linking addition and subtraction word problems and symbols, Journal for Research in Mathematics Education, 22, pp. 266-280.

CARPENTER, T.P., HWEBERT, J. y MOSER,J.M., 1981.Problem structure and first-grade children's initial solution processes for simple addition and subtraction problems, Journal for Research in Mathematics Education, 12, pp. 27-39.

CARPENTER, T. P. y MOSER, J. M., 1982. The development of addition and subtraction problem-solving skills, en T.P. Carpenter, J. M. Moser y T. A. Romberg (eds.), Addition and subtraction: A cognitive perspective, pp, 9-24. (Erlbaum: Hillsdale, NJ).

CARPENTER, T. P. y MOSER, J. M., 1983. The acquisition of addition and subtraction concepts, en $R$. Lesh y M. Landau (eds.), Adquisitions of Mathematics Concepts and Processes, pp. 7-44. (Academic Press.: Orlando, Ftorida).

CARPENTER, T. P. y MOSER, J. M., 1984, The acquisitions 
of addition and subtraction concepts in grades one through three, Journal for Research in Mathematics Education, is, pp. 179-202.

CARPENTER, T. P., MOSER, J. M. y ROMBERG, T. A. (eds.), 1982. Addition and Subtraction: A Cognitive Perspective. (Erlbaum: Hillsdale, NJ).

CARPENTER, T. P., MOSER, J. M. y BEBOUT, H. C., 1988. Representation of addition and subtraction word problems, Journal for Research in Mathematics Education. 19, pp. 345-357.

CASTRO, E.,RICO,L., BATANERO, C. y CASTRO,E., 1991. Dificuliad en problemas de comparacion multiplicativa, en F. Furinghetti (ed.) Proceedings Fifteenth PME Conference. Vol, 1, pp. 192-198. Assisi. Italy.

DAHMUS, M. E., 1970. How to teach verbal problems, School Science and Mathematics, 70, pp, 121- 138 .

DE CORTE, E, y VERSCHAFFEL, L., 1985. Beginning first graders' initial representation of arithmetic word problems, The Journal of Mathematical Behavior, 4, pp. 3-21.

DE CORTE, E. y VERSCHAFFEL, L., 1987. The effect of semantic structure on first graders' strategies for solving addition and subtraction word problem, Journal for Research in Mathematics Education, 18, pp. 363-381.

DE CORTE, E., VERSCHAFFEL, L. y DE WIN, L., 1985. Influence of rewording verbal problems on children's problem representation and solutions, Journal of Educational Psychology, 77(4), pp. 460-470.

DE CORTE, E., VERSCHAFFEL, L. y PAUWELS, A., 1990. Influence of the semantic structure of word problems on second graders' eye movements, Journal of Educational Psychology, 82(2), pp. 359-365.

FAYOL, M. y ABDI, H., 1986. Impact des formulations sur la résolution de problèmes additifs chez I'enfant de 6 a 10 ans, European Journal of Psychology of Education, 1(1), pp. 4I. 58.

GOLDIN, G. A. y MCCLINTOCK, C. E. (eds.), 1980. Task variables in mathematical problem solving, pp. 23-68. (The Franklin Institute Press: Philadelphia, Pensilvania).

GONZÁLEZ, E., GUTIÉRREZ, J., RICO, L. y TORTOSA, A., 1985. Relación verbo-operación en los problemas de aritmética del Tercer Ciclo de la EGB. Actas de las IIJornadas Andaluzas de Profesores de Matematicas. Almería. Sociedad Andaluza de Profesores de Matemáticas Thales.

GROWS, D. A., 1972. Open sentences: Some instructional considerations from research, The Arithmetic Teacher, 19 , pp. 595-599.

GROWS, D. A.,1974. Solution methods used in solving addition and subtration open sentences, The Arithmetic Teacher, 21 , pp. 255-261.

HELLER, J. I. y GREENO, J. G., 1979. Information processing analyses of mathematical problem solving, en $R$. Lesh, $D$. Mierkiewicz y M. Kantowskì (eds), Applied Mathematical Problem Solving. (ERIC/SMEAC: Columbus, Ohio).

HIEBERT, H., 1982. The position of the unknown set and children's solutions of verbal probiems, Journal for Research in Mathematics Education, 13(5), pp. 341-349.
JERMAN, M., 1973. Problem length as a structural variable in verbal arithmetic problems, Educotional Studies in Mathematics, 5, pp. 109-123.

JERMAN, M. E. y MIRMAN, S., 1974. Linguistic and computational variables in problem solving in elementary mathematics, Educational Studies in Mathematics, 5, pp. 317-362.

JERMAN, M. y REES, R., 1972. Predicting the relative difficulty of the verbal arithmetics problems, Educational Studies in Mathematics, 4, pp. 306-323.

KANE, R. B., 1970. The readability of mathematics texbooks revisited, The Mathematics Teacher, 63, pp. 579-581.

KILPATRICK,J., 1978. Variables and methodologies in research on problem solving, en L. L. Hatfield y D. A. Bradbard, (eds.), Mathematical problem solving : papers froma research workshop. (ERIC/SMEAC: Columbus, Ohio).

KILPATRICK, J., 1991. A history of research in mathematics education, en D. A. Grows (ed.), Handbook of research on mathematicsteaching and learning. (Macmillan: Nueva York).

KINTSCH, W. y GREENO, J. G., 1985. Understanding and solving word arithmetic problems, Psychological Review, 92, pp. $109-129$

LINDVALL, C. M. eIBARRA, C. G., 1980. Incorrect procedures used by primary grade pupils in solving open addition and subtraction sentences, Journal for Research in Mathematics Education, 11, pp. 50-62.

LINVILLE, W. J., 1976. Syntax, vocabulary, and the verbal arithmetic problem, School Science and Mathematics, 76, pp. $152-158$.

MOYER, J. C., MOYER, M.B., SOWDER, L. Y THREADGILLSOWDER, J.,1984a. Story problem formats: Verbal versus telegraphic, Journal for Research in Mathematics Education, 15 , pp. 64-68.

MOYER,J.C., MOYER, M.B., SOWDER, L. y THREADGILLSOWDER, J., 1984b. Story problem formats: Drawn versus verbal versus telegraphic, Journal for Research in Mathematics Education, 15, pp. 342-351.

NESHER, P., 1976. Three deteminants of difficutty in verbal arithmetic problems, Educational Studies in Mathematics, 7, pp. $369-388$

NESHER, P., 1982. Levels of description in the analysis of addition and subtraction word problems, en T. P. Carpenter, J. M. Moser y T. A. Romberg (eds.), Addition and subtraction: A cognitive perspective, pp. 25-38. (Lawrence Erlbaum Associates: Hillsdale, New Jersey).

NESHER, P. y TEUBAL, E., 1975. Verbal cues as an interfering factor in verbal problem solving, Educational Studies in Mathematics, 6, pp. 41-51.

NESHER, P., GREENO, J. G. y RILEY, M. S., 1982. The development of semantic categories for addition and subtraction, Educational Studies in Mathematics, 13, pp. 373-394.

PAUL, D. J., NIBBELINK, W. H. y HOOVER, H.D., 1986. The effects of adjusting readability on the diffictily of mathematics story problems, Journal for Research in Matematics Education, 17 , pp. $163 \cdot 171$. 
PUIG, L. y CERDÁN, F., 1989.Problemas aritméticos escolares. (Síntesis: Madrid).

REED, H. B., 1949. Psicologia de las materias de enseñanza primaria. (UTEHA: Méxíco).

RICO, L. et al., 1988. Didactica activa para la resolución de problemas. Departamento de Didactica de la Matemática. Universidad de Granada.

RILEY, M. S., GREENO, J. G. y HELLER, J. I,, 1983. Development of children's problem-solving ability in arithmetic, en H. Ginsburg (ed.), The Development of Mathematical Thinking, pp. 153-196. (Academic Press: Orlando, FL.).

STRETCH, L. B., 1941. One hundred selected research studies, en Arithmetic in General Education, pp. 318-327. Sixteenth Yearbook. (NCTM: Nueva York).

SEARLE, B., LORTON, P. y SUPPES, P., 1974. Structural variables affecting CAI performance on arithmetic word problems of disadvantaged and deaf students, Educational Studies in Mathematics, 5, pp. 371-384.

SUPPES,P., LOFTUS, E. y JERMAN, M., 1969. Problem-Solving on a computer-based teletype, Educational Studies in Mathematics, 2, pp. 1-15.

VERGNAUD, G. y DURAND, C., 1976. Structures additives et complexité psicogénétique, Revue Française de Pédagogie, 36 , pp. $28-43$.

VERGNAUD, G., 1982. A classification of cognitive task and operations of thought involved in addition and subtraction problems, en T. P. Carpenter, 3. M. Moser y T. A. Romberg (eds.), Addition and subtraction: A cognitive perspective $\mathrm{pp}$. 39-59. (Lawrence Erlbaum Associates: Hillsdale, New Jersey).

VERGNAUD, G., 1983. Multiplicative structures, en R. Lesh y M. Landau (eds.), Acquisitions of mathematics concepts and processes. (Academic Press: Londres).

WEAVER, J. F., 1971. Some factors associated with pupils' perfomance levels on simple addition and subtraction sentences, The Arithmetic Teacher, 18, pp. 513-519.

WEAVER, J. F., 1972. The ability of first-, second- and thirdgrade pupils to identify open addition and subtraction sentences for which no solution exists within the set of whole numbers, School Science and Mathematics, 72, pp. 679-691.

WEAVER, J. F., 1973. The symetric property of the equality relation and young children's ability to solve open addition and subtraction sentences, Journalfor Research in Mathematics Education, 4, pp. 45-56.

WEBB, N. L., 1980. Content and context variables in problem tasks, en Goldin y McClintock (eds.), 1984. Task Variables in Mathematical Problem Solving . (The Franklin Institute Press: Philadeiphia, Pensilvania). 